\title{
Miranda
}

Revue pluridisciplinaire du monde anglophone /

Multidisciplinary peer-reviewed journal on the English-

speaking world

$18 \mid 2019$

Guerre en poésie, poésie en guerre

\section{Du roman à la scène : Les Noms et Mao II de Don DeLillo vus par Julien Gosselin}

\section{Critique}

\section{Aliette Ventéjoux}

\section{(2) OpenEdition}

Journals

Édition électronique

URL : http://journals.openedition.org/miranda/18086

DOI : 10.4000/miranda.18086

ISSN : 2108-6559

Éditeur

Université Toulouse - Jean Jaurès

Référence électronique

Aliette Ventéjoux, "Du roman à la scène : Les Noms et Mao // de Don DeLillo vus par Julien Gosselin », Miranda [En ligne], 18 | 2019, mis en ligne le 17 avril 2019, consulté le 16 février 2021. URL : http:// journals.openedition.org/miranda/18086 ; DOI : https://doi.org/10.4000/miranda.18086

Ce document a été généré automatiquement le 16 février 2021.

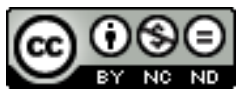

Miranda is licensed under a Creative Commons Attribution-NonCommercial-NoDerivatives 4.0 International License. 


\section{Du roman à la scène : Les Noms et Mao II de Don DeLillo vus par Julien Gosselin}

Critique

Aliette Ventéjoux

\section{Informations sur la pièce}

1 Lieu: Odéon Théâtre de l'Europe - Ateliers Berthier. 13 décembre 2018, 20 h et 19 décembre 2018, $20 \mathrm{~h}$.

2 Metteur en scène : Julien Gosselin

3 Comédiens, comédiennes: Rémi Alexandre, Guillaume Bachelé, Adama Diop, Joseph Drouet, Denis Eyriey, Antoine Ferron, Noémie Gantier, Carine Goron, Alexandre LecrocLecerf, Frédéric Leidgens, Caroline Mounier, Victoria Quesnel, Maxence Vandevelde Compagnie : Si vous pouviez lécher mon cœur

5 Traduction : Marianne Véron

6 Scénographe : Hubert Colas

7 Assistant à la mise en scène : Kaspar Tainturier-Fink

8 Création musicale : Rémi Alexandre, Guillaume Bachelé, Maxence Vandevelde

9 Création lumière : Nicolas Joubert assisté d'Arnaud Godest

10 Création vidéo : Jérémie Bernaert, Pierre Martin

11 Création sonore : Julien Feryn

12 Costumes : Caroline Tavernier assistée d'Angélique Legrand

13 Accessoires : Guillaume Lepert 


\section{Liens}

fois-ci à l'œuvre de DeLillo, plus particulièrement à trois romans, Joueurs, Mao II et Les Noms, qui ont été présentés pour la première fois à Avignon en 2018. Ils étaient joués en novembre et décembre aux Ateliers Berthier à Paris. La trilogie y était donnée le weekend, et les pièces seules pouvaient être vues en soirée durant la semaine. N'ayant pas eu la chance de pouvoir assister à la «représentation marathon» (plus de 9h d'affilée prévues pour les trois pièces) du samedi 8 décembre qui a été annulée-pour des raisons qui n'étaient en rien liées au spectacle-j'ai pu me procurer des places pour les représentations auxquelles je pouvais encore assister et ai pu voir Les Noms et Mao II, dont il sera ici question.

Les fils conducteurs entre les romans choisis par Gosselin, et traduits en français par Marianne Véron, sont multiples, mais les plus frappants restent le la violence et le langage. Dans Les Noms (1982), une secte mystérieuse assassine ses victimes de manière à ce que leurs initiales correspondent à celles du lieu où elles sont exécutées (par exemple, Michaelsis Kalliambetsos est tué dans un village dont le nom est Mikro Kamini). Le nom de la secte n'est autre que Ta Onómata, qui signifie «les noms » en grec. Mao II dresse le portrait d'un écrivain qui a décidé de se retirer du monde mais qui finit par sortir de sa retraite et se retrouve en prise avec le terrorisme des années 1990 au Moyen-Orient. Si, au début du texte, une photographe vient jusqu'à lui pour le prendre en photo, c'est déjà pour souligner le pouvoir du langage des images qui prend une ampleur certaine dans la mise en scène.

Dans les deux pièces, le spectacle n'advient pas seulement sur scène, mais aussi sur un écran qui domine la scène, disparaît parfois pour ensuite réapparaître et se substituer à celle-ci. Les comédien-ne-s sont filmé-e-s en permanence, et ce jeu de caméra, s'il peut sembler déroutant au début, s'intègre parfaitement à la mise en scène des pièces. D'après les concepteur et réalisateur vidéo, Pierre Martin et Jérémie Bernaert, "cette présence de la vidéo s'est imposée de manière organique parce que le travail sur l'image permet paradoxalement de restituer la qualité purement littéraire des textes ${ }^{1}$.

Par l'usage de l'écran, Gosselin questionne la place de l'image dans notre société, le rôle qu'elle joue désormais, et force le spectateur à se demander si ce rôle est semblable à celui du langage, ou s'il lui est devenu supérieur. Tel un shrapnel organique ${ }^{2}$, pour reprendre une expression DeLillo lui-même, les images sont aujourd'hui partie intégrante de notre société. C'est en effet la sensation dont le public fait l'expérience, étant happé par les différentes images qui s'immiscent en lui, comme le shrapnel, qu'elles soient projetées sur l'écran, tel un film, ou qu'elles transparaissent à travers les comédien-ne-s. Si le choix du metteur en scène de laisser une place si importante à l'écran peut tout d'abord étonner le public, et que nous pouvons parfois nous demander où se trouve la frontière entre cinéma et théâtre, ce quatrième mur dressé entre le public et la troupe ne remet pas en question le caractère théâtral des pièces. En effet, 
même si la troupe passe parfois de longs moments hors-scène, choix inhabituel pour le théâtre, la musique, la fumée, l'odeur de cigarette, ou les ombres de leurs corps, nous rappellent qu'ils sont toujours là et n'ont pas été effacés par l'écran. Ce choix signale par ailleurs la prédominance de l'objet télévision dans les années où les romans de DeLillo ont été écrits ${ }^{3}$, et permet de poursuivre le questionnement lancé par l'auteur sur la puissance totale de la télévision et des images, au détriment de l'écrit et des mots. Se pose également la question du pouvoir de la littérature dans nos sociétés où violence, terreur et images vont désormais de concert.

Le début de Mao II est surprenant pour qui est un peu familier du texte de DeLillo : le roman s'ouvre en effet sur deux photos de foules ${ }^{4}$, puis sur la description des mariés « en masse» de la secte Moon, dans un stade. Au contraire, la pièce débute avec l'apparition sur l'écran d'une jeune femme en robe de mariée, alors que sur la scène la comédienne qui interprête Brita explique ce qui arrive à cette jeune mariée.

21 Si Gosselin fait le choix du particulier par rapport au collectif, l'urgence du texte, les différentes langues parlées par la comédienne Carine Goron, ainsi que la musique et la voix de cette dernière, toujours plus fortes, font en sorte de donner l'impression au spectateur qu'une foule est belle et bien présente, comme dans le Yankee Stadium mentionné au début du roman. Le public est happé dans l'urgence du texte, qu'il comprenne ou non les langues dans lesquelles s'exprime la jeune femme.

L'écran devient ensuite un écran d'ordinateur, sur lequel le texte est écrit au fur et à mesure. Des erreurs apparaissent, qui sont corrigées. Un peu plus loin, dans une mise en abyme de l'acte d'écriture, le metteur en scène fait apparaître sur l'écran les mots que l'écrivain trace sur une feuille, assis à son bureau sur scène. L'acte d'écriture devient alors visible sur la page et sur l'écran, se révélant au spectateur devenu lecteur, dans un premier basculement de l'image vers le texte. L'écran peut enfin être parfois simplement une page, sur laquelle apparaît un nom, comme les noms qui annoncent les différentes parties des deux romans.

Une scène m'a particulièrement interpellée, celle où Brita (la photographe venue pour faire des images de Bill Gray) et Karen (la jeune mariée du début) courent dans le décor (une sorte de cage carrée qui représentent des couloirs dans lesquels des gens courent en sens inverse). La salle est envahie de fumée, et les comédiennes tournent en rond dans une fuite qui n'en finit plus. Cette scène m'a rappelé un passage de Falling Man, autre roman de DeLillo traitant du terrorisme, lorsque Florence Givens raconte sa descente pour s'échapper du World Trade Center. Les couleurs noires et blanches qui dominent cette scène, ainsi que la fumée toujours présente, entretiennent l'impression d'assister à une catastrophe.

Il faut également noter que l'écrivain Bill Gray (joué par Frédéric Leidgens), personnage central du roman, apparaît comme un trait d'union entre le questionnement sur la place de l'image et sur celui de la place du terrorisme dans nos sociétés contemporaines. Il a en effet décidé de se retirer du monde et accepte difficilement qu'une photographe, Brita (jouée par Noémie Garnier) vienne faire son portrait. Par ailleurs, lorsqu'il décide enfin de sortir de sa solitude, c'est pour être finalement victime du terrorisme dont il analyse ainsi la place : «There's a curious knot that binds novelists and terrorists. In the West we become famous effigies as our books lose the power to shape and influence. [...] Years ago I used to think that it was possible for a novelist to alter the inner life of the culture. Now bomb-makers and gunmen have taken that territory. They make raids on human consciousness. What writers used to do 
when they were all incorporated. $\aleph^{5}$ En plaçant ces mots dans la bouche d'un écrivain, DeLillo et Gosselin nous invitent peut-être à nous arrêter sur leur place dans la société contemporaine, rappelant encore et toujours l'importance des noms et de leur tissage, et entre autres celui du nom «terroriste » lui-même, bien souvent utilisé de manière inconsidérée.

Entre Mao II et Les Noms, une nouvelle de DeLillo sert d'interlude. "Le Marteau et la faucille ", court texte datant de $2011^{6}$, soumet le public à une autre forme de violence, financière cette fois-ci. Réflexion sur le capitalisme, cette nouvelle nous abreuve de noms de villes et de places boursières-le nom de Dubaï, répété à l'infini, résonne encore-pendant le monologue époustouflant du comédien Joseph Drouet, seul face à la salle, accompagné lui aussi par la musique lancinante qui, comme sa diction, par moment s'emballe et nous transmet l'urgence qui habite ces places financières. En sortant le public de la problématique du terrorisme, cet interlude se veut sans doute être une respiration, mais il devient rapidement suffocant, car les problèmes qu'il aborde ne sont pas plus réjouissants, bien au contraire. La voix du comédien est traitée, elle a des sonorités presque mécaniques, automatiques, faisant allusion au caractère inhumain des marchés financiers. Quant à ce qui est des couleurs qui restent attachées à ce texte, on ne sera pas étonné de se rappeler du rouge. Dénonciation du capitalisme et de la folie des marchés, d'une autre forme de violence de ce monde, il n'en apparaît pas moins presque hors contexte pour le spectateur qui ne voit pas les trois pièces d'affilée. L'univers proposé est tellement différent que de celui de Mao II que l'on a l'impression d'avoir assisté à une autre pièce, alors qu'on aurait préféré rester encore un peu avec les personnages de Mao II et les sensations procurées par la pièce qui vient juste de s'achever7 . Cela n'enlève malgré tout rien à la puissance du texte, ni à la performance du comédien, et le texte trouve probablement encore plus toute sa place au cours de la trilogie.

Sur les deux pièces vues, et si toutes les deux méritent leur succès, c'est tout de même Les Noms qui m'auront le plus marquée, bousculée, touchée.

Le titre l'annonce, et le spectacle le montre: le sujet du texte n'est pas seulement le terrorisme, mais bel et bien le langage, l'écriture, les mots eux-mêmes. Qu'ils apparaissent à l'écran ou soient prononcés par les comédien-ne-s, ou déformés par le jeune enfant de Kathryn et James, Tap, qui écrit un roman, les noms sont au cœur de la pièce. Tap n'est pas seulement un écrivain en herbe, il joue aussi avec la langue orale, et parle le « $\mathrm{Ob}$ » avec sa mère. Cela consiste à insérer $o-b$ au cœur des mots : «You insert $o-b$ in certain parts of words. $»^{8} \mathrm{Il}$ résiste ainsi d'une certaine manière à l'oral, inventant une nouvelle forme de langage, mais dans laquelle il est également difficile de ne pas lire le préfixe -ob de "oblitération ». Ce rapport particulier au langage est rendu sur scène par l'absence de l'enfant, dont les mots apparaissent sur l'écran et avec qui les comédien-ne-s jouant ses parents dialoguent, instaurant un lien surprenant entre l'écran-qui devient lui aussi comédien le temps d'une scène en donnant le point de vue de l'enfant-et les deux comédien-ne-s qui se trouvent sur scène, mais aussi entre l'écrit et l'oral. L'écrit de Tap est d'ailleurs lui aussi particulier, comme le démontrent les phrases qui apparaissent sur l'écran et dont la grammaire, l'orthographe et la syntaxe sont pour le moins originales, à l'image du roman qu'il écrit, « a nonfiction novel »9.

Une scène marquante reste sans doute la dispute entre James (Adama Diop) et Kathryn (Victoria Quesnel) qui a lieu durant un changement de plateau : le monde s'agite autour d'eux mais ils ne semblent pas s'en apercevoir et restent tout entier concentrés sur leur 
échange, sur les mots qu'ils prononcent, qui deviennent de plus en plus forts, de plus en plus durs, et parfois même complètement incompréhensibles. Le verbe leur échappe, et échappe au public, perdu dans des hurlements et soulignant l'aspect souvent problématique du langage. Il n'y a pas de répit, pas de temps morts entre les répliques ; les mots fusent. Les comédien-ne-s, quant à eux, sont parfois dos au public sur scène, mais lui font malgré tout parfaitement face grâce à l'intermédiaire de l'écran, comme l'illustre la photo ci-dessous où l'on peut voir Valérie Quesnel.

Fig. 1

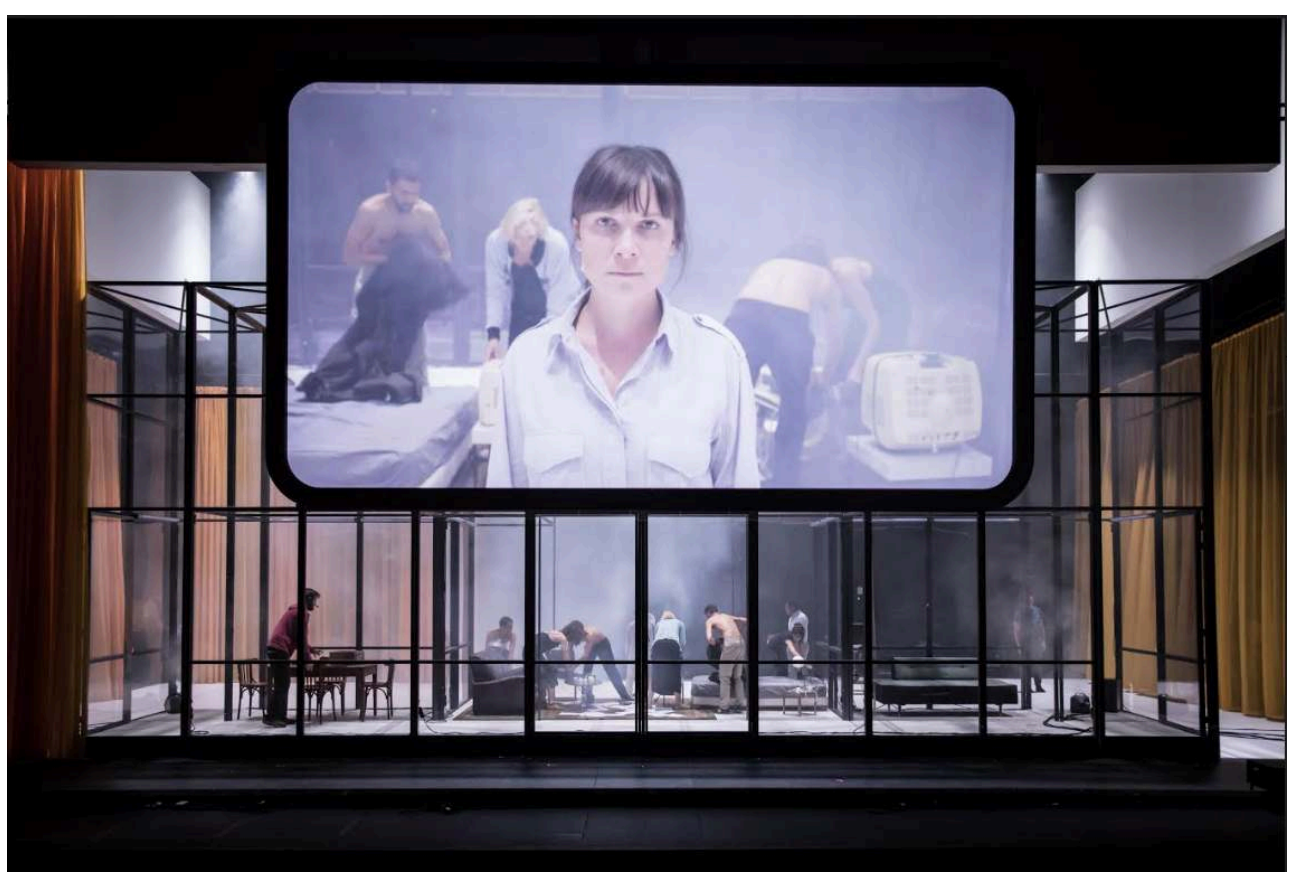

Les Noms, Julien Gosselin.

Crédit @Simon Gosselin

29 Les quelques longueurs du monologue de fin, au cours duquel Owen Brademas (joué par Frédéric Leidgens) revient sur son enfance au sein d'une église des États-Unis, et sur la difficulté de son accès au langage du groupe à cette époque, sont effacées par la toute dernière scène. Les Noms s'achèvent en effet en une glossolalie à couper le souffle, les cris et vociférations des comédien.ne's se mélangent à une basse qui vous prend aux tripes, un sentiment de terreur pure envahi le spectateur qui accepte de se laisser emporter, et assiste impuissant à cette apocalypse qui advient sur scène, rendue encore plus crédible par la pluie qui dégouline sur les comédien-ne's. Trempés, hurlant en toute langue, en transe, ils finissent nus et quasi muets, et il semble que le langage finisse par échapper au sens, plus aucune pensée n'est articulée, et finalement tout s'arrête net.

Si l'on peut parier que la fin de la trilogie marque l'épuisement du spectateur, des comédien'ne·s, des musiciens, des caméramans et techniciens, il est certain que le texte de DeLillo, comme la mise en scène de Gosselin, soulignent que jamais n'advient l'épuisement du verbe. 


\section{NOTES}

1. « Avignon : Julien Gosselin, l'écriture sur un plateau ». Fabienne Darge. Le Monde. 6 juillet 2018. https://www.lemonde.fr/festival-d-avignon/article/2018/07/06/avignon-julien-gosselin-1ecriture-sur-un-plateau_5326795_4406278.html

2. C'est dans son roman Falling Man, que DeLillo introduit l'image du shrapnel organique. Un docteur explique à Keith (un survivant des attaques du 11 septembre), ce qu'est un shrapnel organique : "A student is sitting in a café. She survives the attack. Then, months later, they find these little, like, pellets of flesh, human flesh that got driven into the skin. They call this organic shrapnel." (Falling Man, 16).

3. Players en 1977, The Names en 1982 et Mao II en 1991. La prédominance de la télévision et des images est bien toujours d'actualité, même si les écrants sont aujourd'hui différents de ceux d'alors (tablettes, smartphones, ordinateurs, ...).

4. Nous notons ici que ces photos n'apparaissent pas dans la traduction française.

5. Don DeLillo. Mao II. London : Vintage. 1992 (1991). 41.

6. Cette nouvelle a été publiée dans le recueil The Angel Esmeralda sous le titre "Hammer and Sickle ». Don DeLillo. The Angel Esmeralda. New York : Scribner. 2011. 147-182.

7. La sensation est probablement différente lorsque l'on voit les trois pièces d'affilée.

8. Don DeLillo. The Names. London: Picador. 2011. (1982).

9. Ibid.

\section{RÉSUMÉS}

Compte rendu de deux des pièces de la trilogie (Joueurs, Mao II, Les Noms) adaptée des romans de DeLillo, mis en scène par Julien Gosselin.

Performance review of two of the three plays of the trilogy adapted from Don DeLillo's novels (Players, Mao II, The Names), directed by Julien Gosselin.

\section{INDEX}

Mots-clés : Don DeLillo, écran, image, Julien Gosselin, langage, littérature contemporaine, mots, Si vous pouviez lécher mon cœur, terreur, terrorisme, théâtre

Thèmes : Theater

Keywords : contemporary literature, Don DeLillo, image, Julien Gosselin, language, screen, Si vous pouviez lécher mon cœur, terror, terrorism, theatre 
AUTEUR

\section{ALIETTE VENTÉJOUX}

Université Paris II - Panthéon Assas

alietteventejoux@protonmail.com 